\title{
Efficient $\mathrm{LaF}_{3}: \mathbf{N d}^{3+}$-based vacuum-ultraviolet laser at $172 \mathrm{~nm}$
}

\author{
M. A. Dubinskii, ${ }^{*}$ A. C. Cefalas, E. Sarantopoulou, S. M. Spyrou, and C. A. Nicolaides \\ Theoretical and Physical Chemistry Institute, National Hellenic Research Foundation, \\ 48 Vassileos Constantinou Avenue, Athens 116-35, Greece
}

R. Yu. Abdulsabirov, S. L. Korableva, and V. V. Semashko

Kazan State University, 18 Lenin Street, 420008 Kazan, Russia

Received June 18, 1991

\begin{abstract}
Vacuum-ultraviolet (VUV) laser radiation at $172 \mathrm{~nm}$ has been obtained from a solid-state $\mathrm{LaF}_{3}: \mathrm{Nd}^{3+}$-based laser pumped by a pulsed-discharge molecular $\mathrm{F}_{2}$ laser at $157 \mathrm{~nm}$. The maximum slope efficiency of the solidstate laser described in this experiment was $21 \%$ ( $14 \%$ conversion efficiency), and the maximum output energy at $172 \mathrm{~nm}$ was $0.4 \mathrm{~mJ}$ for a nonoptimized optical cavity. This finding introduces serious prospects for realizing versions of active-medium-plus-source tunable VUV laser devices.
\end{abstract}

\section{INTRODUCTION}

Vacuum-ultraviolet (VUV) laser sources are useful devices for a variety of applications ranging from photochemistry to photolithography. However, until now only a few laser sources (such as $\mathrm{H}_{2}$ and $\mathrm{F}_{2}$ ), which have limited tunabilities, operate in this spectral region. Tunable VUV radiation has been obtained by a variety of methods, such as discharge excitation of atomic beams ${ }^{1}$ and frequency mixing in gases and metal vapors, ${ }^{2,3}$ but tunable VUV radiation can in principle be obtained from a wide class of solid-state active media, such as rare-earth-activated wide-band-gap fluoride dielectric crystals because of the allowed radiative $d-f$ transitions. ${ }^{4}$ Recent developments in new single-crystal wide-band-gap laser materials ${ }^{5-7}$ offer encouraging prospects for the development of crystalbased efficient VUV laser devices. Among the wellknown fluoride crystals $\mathrm{LaF}_{3}: \mathrm{Nd}^{3+}$ (LaF:Nd) now seems to be the most suitable for VUV applications. The simplified energy-level diagram of $\mathrm{Nd}^{3+}$ ions in $\mathrm{LaF}_{3}$ is shown in Fig. 1, and its excitation and emission spectra were studied previously. ${ }^{4,8-10}$ The strong and broad overlapping peaks of the excitation spectra (one of them centered at $159 \mathrm{~nm}$ ) originate from transitions from the $4 f^{3}$ ground state to the crystal field split $4 f^{2} 5 d$ levels of the $\mathrm{Nd}^{3+}$ ion in $\mathrm{LaF}_{3}$. However, among the five possible transitions that have been predicted by theoretical considerations concerning the $\mathrm{C}_{2}$ site symmetry of the $\mathrm{Nd}^{3+}$ in $\mathrm{LaF}_{3}$, only four were observed. ${ }^{4}$ The observed VUV fluorescence originates from the $4 f^{2} 5 d \rightarrow 4 f^{3}$ electric dipole-allowed transitions. They have been assigned to the $5 d \rightarrow{ }^{4} I_{11 / 2}$ and $5 d \rightarrow{ }^{4} I_{13 / 2}$ interconfigurational transitions. ${ }^{8}$ The fluorescence spectrum seems to have a simple structure at room temperature, ${ }^{9}$ and the VUV quantum yield of the $\mathrm{Nd}^{3+}$ ions in the $\mathrm{LaF}_{3}$ host is more than $50 \% .^{8}$ The depression of the $4 f^{2} 5 d$ levels in $\mathrm{LaF}_{3}$ caused by the crystal field is the lowest among the known fluorides, and the onset of the $4 f^{2} 5 d$-configuration levels is observed at frequencies as high as $60000 \mathrm{~cm}^{-1}$. Therefore, the $\mathrm{LaF}: \mathrm{Nd}$ laser should have one of the shortest wavelengths of all possible $\mathrm{Nd}^{3+}$-activated VUV lasers, provided that the appropriate pumping is available. Direct evidence of VUV laser action in LaF: Nd was obtained recently, ${ }^{9,10}$ and this was the first solid-state laser in the VUV region of the spectrum. However, the method of excitation of the active medium that was used in Refs. 9 and 10 (the excitation of the active medium was due to fluorescence of $\mathrm{Kr}_{2}{ }^{*}$ caused by an electron beam) was too complicated to be widely used at the laboratory scale. Therefore a simple and reliable pumping source could enhance the use of these sources for spectroscopic and other applications. Recently ${ }^{11}$ we obtained laser action at $172 \mathrm{~nm}$ from $\mathrm{LaF}_{3}: \mathrm{Nd}^{3+}$ crystals pumped by a molecular $\mathrm{F}_{2}$ laser at $157 \mathrm{~nm}$ with a $3 \%$ conversion efficiency. In this paper we report the observation of a $14 \%$ conversion efficiency with a $21 \%$ slope efficiency [defined as the $\tan \left(\Delta E_{o} / \Delta E_{i}\right)$, where $\Delta E_{o}$ is the change of the output energy when the input energy changes by $\Delta E_{i}$ near threshold] laser action at $172 \mathrm{~nm}$ from a LaF : Nd crystal transversely pumped by a molecular $\mathrm{F}_{2}$ laser at $157 \mathrm{~nm}$. The energy conversion of this system is determined, and the processes that limit the energy available from the crystal are discussed.

\section{EXPERIMENT}

The $\mathrm{LaF}: \mathrm{Nd}$ crystals used in this experiment were grown from carbon crucibles by the Bridgman-Stockbarger method. Because the VUV transparency of the samples depends strongly on the amount and the types of impurities, during the growth process all possible methods of initial products and growth atmosphere refining were used. The laser samples, cylindrical rods $35 \mathrm{~mm}$ long with 5-mm radii, were pumped transversely, and the ends of the laser rods were optically flat and parallel with $10 \mathrm{~s}$ of arc. A polished flat window was made on the side of the cylindrical surface of the crystal for efficient excitation by the pumping laser source. The concentrations of the $\mathrm{Nd}^{3+}$ ions used in these crystals were between $0.3 \%$ 


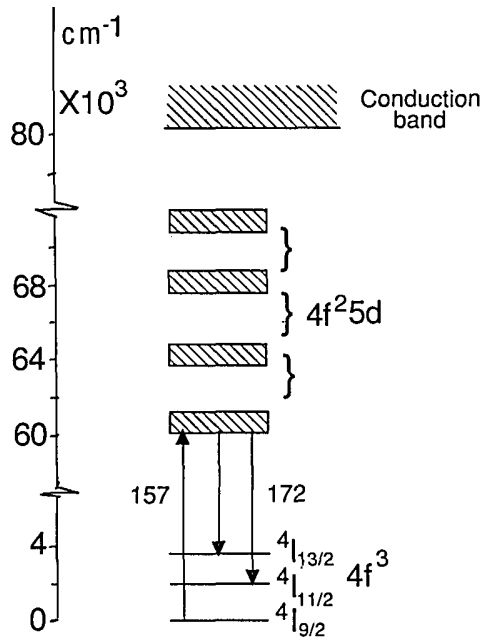

Fig. 1. Energy diagram of $\mathrm{LaF}_{3}: \mathrm{Nd}^{3+}$; the absorption at $157 \mathrm{~nm}$ and the laser emission transitions at $172 \mathrm{~nm}$ are indicated between the $4 f^{3}$ and $4 f^{2} 5 d$ levels.

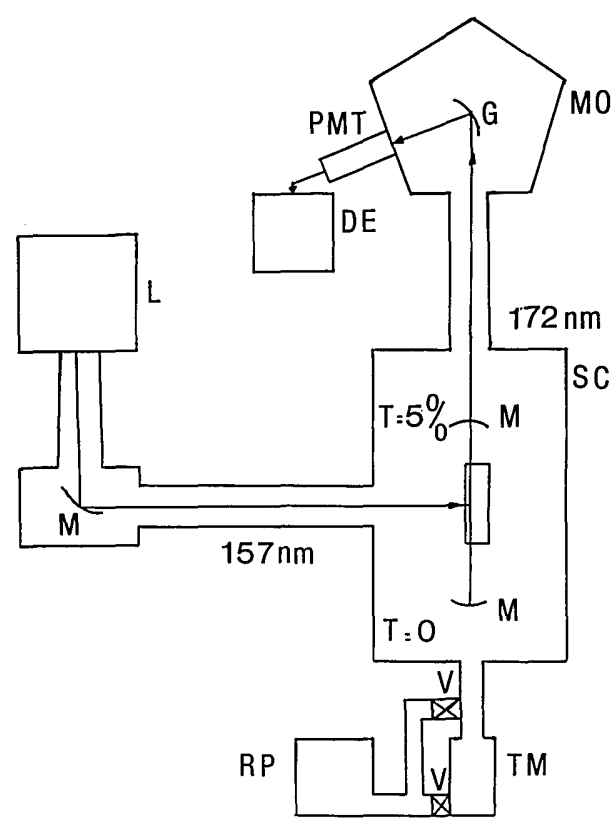

Fig. 2. Experimental layout. $\mathrm{L}$, pulsed-discharge $\mathrm{F}_{2}$ molecular laser at $157 \mathrm{~nm}$; M's, mirrors; SC, stainless-steel vacuum chamber; MO, monochromator; G, grating; PMT, photomultiplier; $\mathrm{DE}$, detection electronics; TM, turbo molecular pump; RP, rotary pump; V's, valves.

and 1\%. The experimental apparatus is shown in Fig. 2. The pumping source was an $\mathrm{F}_{2}$ molecular laser of the fast-discharge type that has been described previously. ${ }^{12,13}$ The maximum available energy from this device was $12 \mathrm{~mJ}$ at $3.5 \mathrm{~atm}$ (2.7 kTorr) total gas pressure. The pumping laser pulse had a width of $12 \mathrm{~ns}$ at half-maximum (Fig. 3). The pumping laser beam was focused on the crystal with an off-axis concave mirror with a $120-\mathrm{cm}$ radius of curvature. The maximum energy density at the plane of the input window of the crystal was $120 \mathrm{~mJ} / \mathrm{cm}^{2}$ in a rectangular geometry of the focal spot with dimensions $0.3 \mathrm{~mm} \times 35 \mathrm{~mm}$. The solid-state laser cavity was of the stable near-confocal type, and it was $15 \mathrm{~cm}$ long. Both the output and the back mirrors ( $\mathrm{Al}+\mathrm{MgF}_{2}$ deposited on a substrate of $\mathrm{BaF}_{2}$ ) of the cavity had a $30-\mathrm{cm}$ radius of curvature. The back mirror was totally nontransparent at $172 \mathrm{~nm}$, while the transmittance of the output coupler at the same wavelength was $65 \%$. The laser signal was detected with $0.2-\mathrm{m}$ VUV monochromator (Acton VM 502), a solar-blind fast photomultiplier (EMI 9412), and a Tektronix 7104 oscilloscope. Because of strong absorption at these wavelengths by air, the optical path of the pumping and the solid-state laser beams was within stainless-steel vacuum lines pumped down to $10^{-5}$ mbar $\left(7 \times 10^{-6}\right.$ Torr $)$ with a turbomolecular pump. The pumping and the solid-state laser output energies were measured with a Gentek (PR100-type) pyroelectric detector.

\section{RESULTS AND DISCUSSION}

Stimulated emission from two LaF : Nd crystals, pumped by an $\mathrm{F}_{2}$ molecular laser at $157 \mathrm{~nm}$, was obtained. The concentration of the $\mathrm{Nd}^{3+}$ dopant was 0.5 and 1.0 at. $\%$. The pumping thresholds for obtaining stimulated emission from the two crystals were 3 and $0.5 \mathrm{~mJ}$, respectively.

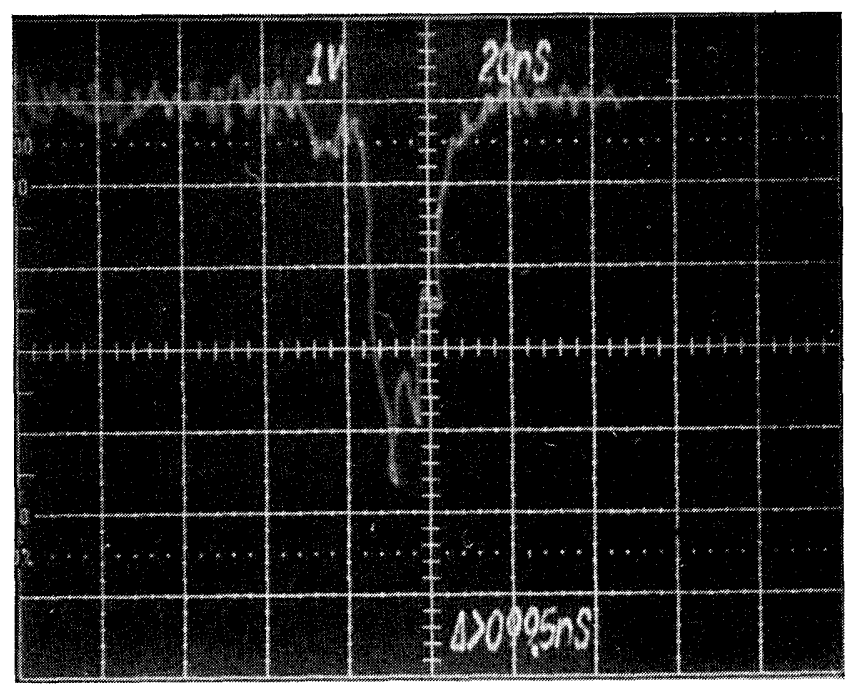

Fig. 3. Temporal evolution of the pumping $F_{2}$ laser pulse at $157 \mathrm{~nm}$.

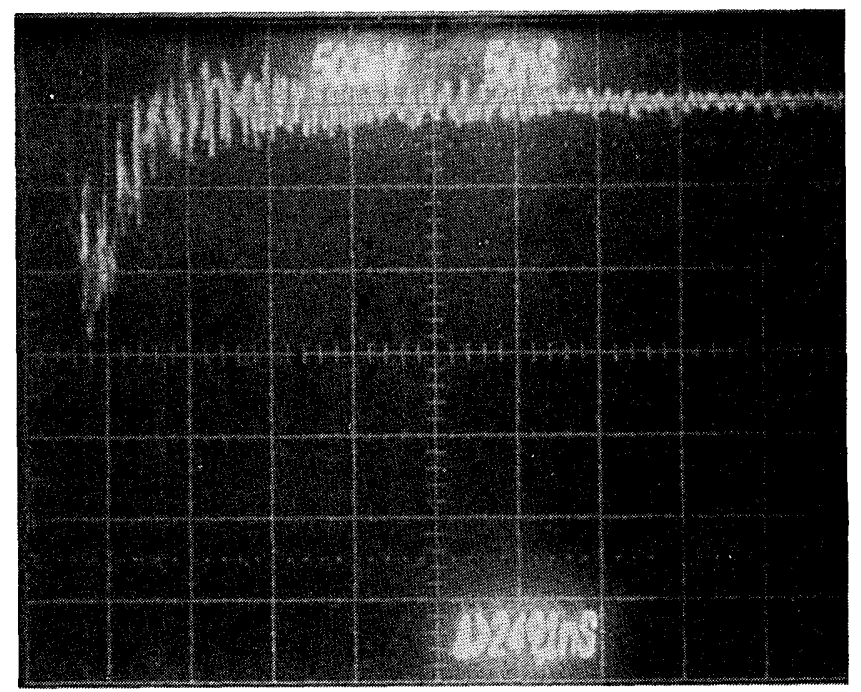

Fig. 4. Temporal evolution of the laser pulse from the $\mathrm{LaF}_{3}: \mathrm{Nd}^{3+}$ crystal at $172 \mathrm{~nm}$. 


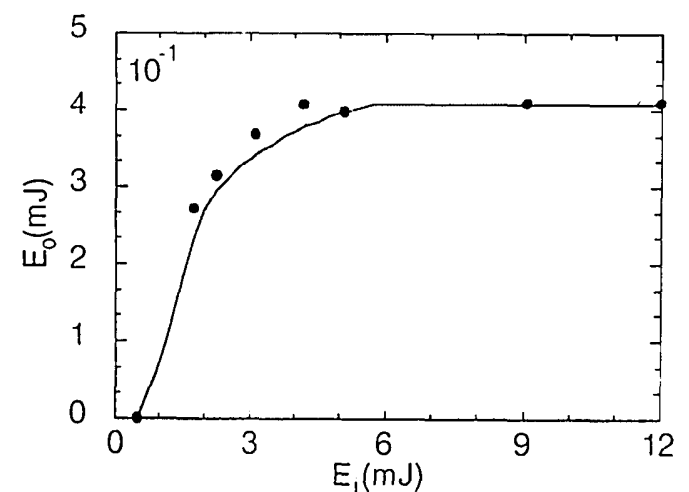

Fig. 5. Output energy $E_{o}$ at $172 \mathrm{~nm}$ from the $\mathrm{LaF}_{3}: \mathrm{Nd}^{3+}$ crystal as a function of the input energy $E_{i}$ of the pumping laser pulse at $157 \mathrm{~nm}$

Stimulated emission from a LaF : Nd crystal was observed previously by Waynant ${ }^{9,10}$ for an only $0.1 \%$ concentration of the $\mathrm{Nd}^{3+}$. The differences between our and Waynant's data are attributed to the different pumping geometries. The transverse pumping geometry of our experiment requires the active medium to have higher coefficients of absorption of the pumping radiation, which are unattainable at low doping levels. The temporal evolution of the generated pulse at $172 \mathrm{~nm}$ is indicated in Fig. 4. The pumping energy was 1.3 times threshold. Because the two pulses have similar temporal evolutions, the exhibition of high gain by the crystal is expected. The output energy of the LaF:Nd VUV laser as a function of the pumping energy is indicated in Fig. 5. The slope efficiency of the described experimental setup for the linear part of the curve (Fig. 5) was $21 \%$. Saturation is also taking place, while the mechanisms of this process are probably similar to those that occur for a YLF : $\mathrm{Ce}^{3+}$-laser, ${ }^{14}$ such as the formation of transient color centers by excitedstate absorption of the pumping radiation. ${ }^{15,16}$ The presence of constant visible coloration of the active media was not observed for a 5-h irradiation of the crystal by the VUV $\mathrm{F}_{2}$ laser and with a $2-\mathrm{Hz}$ repetition rate. Despite the presence of saturation processes in the active media, the maximum output energy at $172 \mathrm{~nm}$ from the excited crystal was $0.4 \mathrm{~mJ}$ for a nonoptimized optical cavity. The low-threshold energy flux and the linear part of Fig. 5 indicate the presence of high gain in the crystal.

\section{CONCLUSIONS}

Vacuum-ultraviolet (VUV) laser radiation has been obtained from a solid-state $\mathrm{LaF}_{3}: \mathrm{Nd}^{3+}$ laser at $172 \mathrm{~nm}$ pumped by a molecular $\mathrm{F}_{2}$ laser at $157 \mathrm{~nm}$. The system exhibits an extremely high gain. Therefore good prospects become apparent for further developments of solidstate VUV tunable lasers based on these crystals with $\mathrm{F}_{2}$ laser pumping. Studies of other $\mathrm{Nd}^{3+}$-activated, as well as other rare-earth-ion-activated fluoride single crystals with the same pumping for UV and VUV laser development are in progress.

\section{ACKNOWLEDGMENTS}

M. Dubinskii thanks the National Hellenic Research Foundation for financial support and hospitality during the time of the experiments. We acknowledge stimulating discussions with C. Webb and S. Hooker, Oxford University, U.K., who are working on a similar project.

*Permanent address, Kazan State University, 18 Lenin Street, 420008 Kazan, Russia.

\section{REFERENCES}

1. T. Efthimiopoulos, B. P. Stoicheff, and R. I. Thomson, Opt. Lett. 14, 624 (1989).

2. M. Jamroz, P. E. La Rocque, and B. P. Stoicheff, Opt. Lett. 7, 617 (1982).

3. J. C. Miller, R. N. Compton, and C. P. Cooper, J. Chem. Phys. 76, 3967 (1982).

4. K. H. Yang and J. A. De Luca, Appl. Phys. Lett. 29, 499 (1976).

5. M. A. Dubinskii, B. N. Kazakov, and Sh. I. Yagudin, Sov. J. Opt. Spectrosc. 63, 698 (1987).

6. R. Yu. Abdulsabirov, M. A. Dubinskii, B. N. Kazakov, N. I. Silkin, and Sh. I. Yagudin, Sov. Phys. Crystallogr. 32, 951 (1987).

7. M. A. Dubinskii, N. M. Khaidukov, I. G. Garipov, L. N. Dem'yanets, A. K. Naumov, V. V. Semashko, and V. A. Malyusov, J. Mod. Opt. 37, 1355 (1990).

8. L. I. Devyatkova, P. M. Lasovskii, V. V. Mikhailin, S. P. Chernov, A. V. Shepelev, and P. B. Esselbakh, Usp. Fiz. Nauk 126, 696 (1978).

9. R. W. Waynant, Appl. Phys. B 828, 205 (1982).

10. R. W. Waynant and P. H. Klein, Appl. Phys. Lett. 46, 14 (1985).

11. M. A. Dubinskii, A. C. Cefalas, and C. A. Nicolaides, Opt. Commun. 88, 122 (1992).

12. C. Scordoulis, E. Sarantopoulou, S. M. Spyrou, and A. C. Cefalas, J. Mod. Opt. 37, 501 (1990).

13. C. Scordoulis, S. M. Spyrou, and A. C. Cefalas, Appl. Phys. B 51, 141 (1990).

14. D. J. Ehrlich, P. F. Moulton, and R. M. Osgood, Opt. Lett. 4, 184 (1979).

15. K. S. Lim and D. S. Hamilton, J. Lumin. 40, 319 (1988).

16. K. S. Lim and D. S. Hamilton, J. Opt. Soc. Am. B 6, 1401 (1989). 